\title{
Aspergillus culture filtrates and sputum sols from patients with pulmonary aspergillosis cause damage to human respiratory ciliated epithelium in vitro
}

\author{
R. Amitani, T. Murayama, R. Nawada, W.J. Lee, \\ A. Niimi, K. Suzuki, E. Tanaka, F. Kuze
}

Aspergillus culture filtrates and sputum sols from patients with pulmonary aspergillosis cause damage to human respiratory ciliated epithelium in vitro. R. Amitani, T. Murayama, R. Nawada, W.J. Lee, A. Niimi, K. Suzuki, E. Tanaka, F. Kuze. @ERS Journals 1995. ABSTRACT: Aspergillus species frequently colonize lower respiratory tracts and lungs with localized underlying conditions (healed tuberculous cavity, cystic fibrosis, bronchiectasis, etc.) even in subjects without systemic predisposing factors.

We investigated the in vitro effects of culture filtrates of Aspergillus species and sputum sols from patients with pulmonary aspergillosis on ciliary beat frequency (CBF) and epithelial integrity of human respiratory ciliated epithelium. Culture filtrates of 25 clinically isolated fungi (16 Aspergillus fumigatus, three Aspergillus niger, one Aspergillus flavus, three Candida albicans, and two Cryptococcus neoformans) were obtained by culturing the fungi in Medium-199 at $37^{\circ} \mathrm{C}$ for 7 days, and five sputum sols were obtained from patients with pulmonary aspergillosis infected by $A$. fumigatus.

During $6 \mathrm{~h}$ experiments using a photometric technique, 14 out of 16 A. fumigatus culture filtrates caused progressive and significant reduction in CBF associated with marked epithelial disruption, whilst the culture filtrates of $A$. niger and $A$. flavus caused minor epithelial damage without slowing of CBF, and Medium-199 alone (Control) showed neither epithelial damage nor slowing of CBF. All of the sputum sols also caused significant slowing of $\mathrm{CBF}$ as well as epithelial disruption. Culture filtrates of $\boldsymbol{C}$. albicans and $\boldsymbol{C r}$. neoformans had no effects on human respiratory epithelium.

We conclude that Aspergillus species, especially A. fumigatus release a factor (or factors) which causes damage to respiratory epithelium and slows CBF, and that these factors may contribute to the colonization of the lower respiratory tracts by the Aspergillus species and may possibly contribute to the further proliferation and spread of the lesions in pulmonary aspergillosis.

Eur Respir J., 1995, 8, 1681-1687.
Dept of Infection and Inflammation, Chest Disease Research Institute, Kyoto University, Sakyo-ku, Kyoto, Japan.

\section{Correspondence: R. Amitani,}

Dept of Infection and Inflammation

Chest Disease Research Institute

Kyoto University

Sakyo-ku

Kyoto 606

Japan.

Keywords: Aspergillus

Aspergillus fumigatus

aspergillosis

respiratory epithelium

cilia

mucociliary clearance

Received: September 301994

Accepted after revision April 141995
Aspergillus species are widely distributed as saprophytic pathogens, which are rarely found in the lower respiratory tract in the general population in which both systemic and localized host defences are intact [1]. However, Aspergillus species frequently colonize the lower respiratory tracts of patients with localized underlying bronchopulmonary conditions, such as healed tuberculosis cavities, cystic fibrosis, bronchiectasis, which are probably associated with impairment of localized host defences, including mucociliary clearance of airways, even in patients without compromised systemic host defences [2-9]. Whereas, other pathogenic fungi, including Candida albicans, Cryptococcus neoformans and Mucor, rarely colonize the lower respiratory tracts in similar conditions.

Several kinds of bacteria, including Pseudomonas aeruginosa, Streptococcus pneumoniae and Haemophilus influenzae, are known to more frequently colonize the lower respiratory tracts in similar localized conditions $[10,11]$, and it is already known that these bacteria release cilio-inhibitory substances, which probably contribute to their continuous colonization by perturbing the mucociliary clearance in the respiratory tracts [12-18].

Although there have so far been no reports, to our knowledge, on the effect of Aspergillus species on human respiratory ciliated epithelium, we have speculated that some of the Aspergillus species also release cilio-inhibitory factor(s) in order not to be overcome by mucociliary clearance, which is a first line defence against invasive pathogens in the respiratory tracts. Thus, the purpose of the present study was to evaluate the ability of Aspergillus species to damage the respiratory ciliated epithelium, by investigating the effects of Aspergillus culture filtrates and sputum sols from patients with pulmonary aspergillosis on ciliary beating and epithelium integrity of human respiratory ciliated epithelium in vitro. 


\section{Materials and methods}

\section{Isolation of fungal strains}

Twenty consecutive clinical isolates of Aspergillus species (16 strains of $A$. fumigatus, three strains of $A$. niger, and one of $A$.flavus) were originally obtained from sputum of patients with pulmonary aspergillosis in the Chest Disease Research Institute, Kyoto University, between 1991 and 1993. C. albicans (three strains) was clinically isolated from patients with oral candidiasis and $\mathrm{Cr}$. neoformans (two strains) from patients with pulmonary cryptococcosis.

\section{Preparation of fungal culture filtrates}

After growth on slants of potato dextrose agar (PDA) (Difco Laboratories, Detroit, USA) for 5 days at $37^{\circ} \mathrm{C}$, conidia $\left(2 \times 10^{7}\right)$ of the Aspergillus species described above were taken from each slant, and cultured in flasks containing $300 \mathrm{~mL}$ of Medium-199 cell culture medium (Gibco Laboratories, Grand Island, NY, USA) without shaking, at $37^{\circ} \mathrm{C}$ for 7 days. Spores $\left(2 \times 10^{7}\right)$ of the $C$. albicans and $\mathrm{Cr}$. neoformans described above were also taken from each slant, and cultured in flasks containing $300 \mathrm{~mL}$ of Medium199 in the same way. After 7 days of culture, the fungi were removed by passing the culture medium through gauze, and then each culture filtrate was sterilized by MillexGV $0.22 \mu \mathrm{m}$ millipore-filters (Japan Millipore Ltd, Tokyo, Japan) and stored at $-80^{\circ} \mathrm{C}$. Before use in experiments, the $\mathrm{pH}$ of each culture filtrate was measured with $\mathrm{F}-22 \mathrm{pH}$ meter (Horiba, Ltd, Kyoto, Japan) ( $\mathrm{pH}$ range of original culture filtrates before adjustment was 8.1-8.3), and adjusted to 7.4 by addition of $1 \mathrm{M} \mathrm{HCl}$ to standardize the ciliary function assay. This adjustment required addition of no more than $0.5 \%$ of the original volume of each culture filtrate. The sterility of the culture filtrates was tested by culturing for three days on PDA plates at $37^{\circ} \mathrm{C}$.

\section{Preparation of sputum sols}

Sputa were collected over $4 \mathrm{~h}$ in sterile pots, as free from saliva as possible, from five patients (Nos. 1-5) without any systemic underlying diseases, who were suffering from pulmonary aspergillosis infected by A. fumigatus. The sputa collected had mucoid or mucopurulent appearance, with the purulent portion being less than about one third. The samples were ultracentrifuged at $100,000 \times \mathrm{g}$ for $60 \mathrm{~min}$ at $4^{\circ} \mathrm{C}$ to obtain the sputum sols. The sputum sols were sterilized by Millex-GV $0.22 \mu \mathrm{m}$ milliporefilters. The $\mathrm{pH}$ of each sputum sol was measured with the $\mathrm{pH}$ meter (range $6.5-8.1$ ) and adjusted to 7.4 by addition of $1 \mathrm{M} \mathrm{HCl}$ or $1 \mathrm{~N} \mathrm{NaOH}$ to standardize the ciliary function assay prior to use in experiments. This adjustment required addition of no more than $0.5 \%$ of the original volume of each sputum sol. Sputa with purulent appearance were collected from four bronchiectatic patients (Nos. P1-P4) infected by $P$. aeruginosa during clinically non-exacerbated status, and was processed in the same way as described above for obtaining sputum sols.

Examination of epithelial damage by light microscopy and measurement of ciliary beat frequency [16, 19]

Human nasal ciliated epithelium was obtained from the inferior turbinate of normal-volunteers by a brushing technique using a cytology brush without local anaesthesia [19]. Strips of ciliated epithelium were dispersed by agitation of the cytology brush in Medium-199. The suspension of ciliated epithelium was divided into four or five equal aliquots, and centrifuged at $200 \times \mathrm{g}$ for 5 min. The supernatant Medium-199 was aspirated and replaced by an equal volume $(400 \mu \mathrm{L})$ of Medium-199 in one sample (Control), and replaced by the test solutions, which consisted of the culture filtrates or the sputum sols of the patients, in the others. Each sample was transferred to a sealed microscope slide-coverslip preparation and kept in an incubator at $37^{\circ} \mathrm{C}$.

Ciliary beat frequency (CBF) was measured at $37^{\circ} \mathrm{C}$ using a photometric technique as reported previously [16]. Ten readings of CBF were taken, from the same epithelial strips of each slide selected at the beginning of the experiment, at $1 \mathrm{~h}$ intervals throughout the $6 \mathrm{~h}$ experiment. The slides were randomized at the beginning of each experiment so that the observer was unaware of the nature of the samples that were tested in each experiment. At each experimental time-point, an assessment of the ciliary beating pattern and the epithelial integrity was made in addition to the measurement of CBF [16]. During each experiment, a note was made of any ciliary dyskinesia (disorganization of normal coordinated beating pattern), ciliostasis (complete cessation of ciliary beating), or epithelial disruption. Epithelial disruption was assessed subjectively by the same investigator and scored from - to +++: - (intact epithelium); + (mild disruption) was undulation of the epithelial strip surface; ++ (moderate disruption) was undulation and extrusion of individual cells from the epithelial strip surface; +++ (severe disruption) was total disorganization of the epithelial strip surface.

\section{Statistical analysis}

The mean CBF of control and test preparations were compared at each time-point, and the maximum percentage of the slowing of test CBF compared to control was identified. At this time-point, the mean CBF $(n=10)$ of test and control preparations were compared using the unpaired Student's t-test.

\section{Results}

\section{Clinical characteristics of patients}

The clinical backgrounds of the patients from whom the fungi were isolated and used in the present study are 
Table 1. - Clinical characteristics of patients from whom fungal strains were isolated

\begin{tabular}{|c|c|c|c|c|c|c|}
\hline $\begin{array}{l}\text { Patient } \\
\text { No. }\end{array}$ & $\begin{array}{l}\text { Fungus } \\
\text { isolated }\end{array}$ & $\begin{array}{l}\text { Strain } \\
\text { No. }\end{array}$ & $\begin{array}{l}\text { Age } \\
\text { yrs }\end{array}$ & Sex & $\begin{array}{l}\text { Fungal } \\
\text { disease }\end{array}$ & $\begin{array}{l}\text { Underlying } \\
\text { conditions }\end{array}$ \\
\hline 1 & A. fumigatus & Af-1 & 44 & $\mathrm{~F}$ & Invasive (CNPA) & Old TB \\
\hline 2 & A. fumigatus & Af- 2 & 64 & $\mathrm{M}$ & Aspergilloma & Pul. emphysema \\
\hline 3 & A. fumigatus & Af-3 & 53 & $\mathrm{~F}$ & Aspergilloma & Bulla \\
\hline 4 & A. fumigatus & Af-4 & 77 & $\mathrm{~F}$ & Invasive (CNPA) & None \\
\hline 5 & A. fumigatus & Af-5 & 75 & M & Aspergilloma & Old TB \\
\hline 6 & A. fumigatus & Af-6 & 56 & M & Invasive (CNPA) & Bulla \\
\hline 7 & A. fumigatus & Af-7 & 72 & M & Invasive (CNPA) & Old TB \\
\hline 8 & A. fumigatus & Af- 8 & 60 & M & Aspergilloma & Old TB \\
\hline 9 & A. fumigatus & Af-9 & 47 & $\mathrm{~F}$ & Aspergilloma & Funnel Chest \\
\hline 10 & A. fumigatus & Af-10 & 62 & M & Invasive & IPF, Steroid (+) \\
\hline 11 & A. fumigatus & Af- 11 & 58 & $\mathrm{M}$ & Invasive & Lung Cancer, Steroid (+) \\
\hline 12 & A. fumigatus & Af- 12 & 73 & $\mathrm{M}$ & Invasive (CPNA) & Old TB \\
\hline 13 & A. fumigatus & Af-13 & 71 & $\mathrm{M}$ & Invasive & IPF, Steroid (+) \\
\hline 14 & A. fumigatus & Af-14 & 53 & $\mathrm{~F}$ & Aspergilloma & Old TB \\
\hline 15 & A. fumigatus & Af- 15 & 30 & $\mathrm{M}$ & Aspergilloma & Old TB \\
\hline 16 & A. fumigatus & Af- 16 & 58 & $\mathrm{~F}$ & Invasive (CNPA) & None \\
\hline 17 & A. niger & An-1 & 60 & $\mathrm{~F}$ & Aspergilloma & Old TB \\
\hline 18 & A. niger & An-2 & 60 & $\mathrm{~F}$ & ABPA & Bronchial Asthma \\
\hline 19 & A. niger & An-3 & 66 & M & Invasive (CNPA) & Old TB \\
\hline 20 & A. flavus & Afl-1 & 62 & M & Invasive (CNPA) & Bronchiectasis \\
\hline 21 & C. albicans & $\mathrm{Ca}-1$ & 50 & $\mathrm{~F}$ & Oral candidiasis & None \\
\hline 22 & C. albicans & $\mathrm{Ca}-2$ & 39 & M & Oral candidiasis & None \\
\hline 23 & C. albicans & $\mathrm{Ca}-3$ & 60 & $\mathrm{~F}$ & Oral candidiasis & None \\
\hline 24 & Cr. neoformans & $\mathrm{Cr}-1$ & 62 & M & Pul. cryptococcosis & None \\
\hline 25 & Cr. neoformans & $\mathrm{Cr}-2$ & 27 & M & Pul. cryptococcosis & None \\
\hline
\end{tabular}

A.: Aspergillus: C.: Candida; Cr.: Cryptococcus; M: male; F; female; Invasive: invasive pulmonary aspergillosis; CNPA: chronic necrotizing pulmonary aspergillosis; Aspergilloma: pulmonary aspergilloma; ABPA: allergic bronchopulmonary aspergillosis; TB: pulmonary tuberculosis; IPF: idiopathic pulmonary fibrosis; Steroid (+): long-term steroid therapy; Pul.: pulmonary. Sputum sols were obtained from patients Nos. 1-5.

shown in Table 1 . Nine of 16 strains of A. fumigatus were isolated from patients with invasive pulmonary aspergillosis, including six cases of chronic necrotizing pulmonary aspergillosis (CNPA) [20], whilst the other strains of $A$. fumigatus were from pulmonary aspergilloma [2, 3]. Each of three strains of A. niger was isolated from a patient with CNPA, pulmonary aspergilloma or allergic bronchopulmonary aspergillosis (ABPA) [21], and the strain of A. flavus was isolated from CNPA. Most of the patients did not have any systemic underlying conditions except for an advanced-staged lung cancer (No. 11) and long-term steroid treatments (Nos. 10, 11, 13). Eighteen out of 20 patients with pulmonary aspergillosis had localized underlying bronchopulmonary disorders, such as healed tuberculosis (nine cases), bulla, pulmonary emphysema, bronchiectasis, and idiopathic pulmonary fibrosis. The other two patients, however, apparently had neither localized nor systemic underlying disorders (Nos. 4 and 16).

The effects of Aspergillus culture filtrates on respiratory ciliated epithelium

Table 2 shows the mean $(n=10)$ CBF values and the degree of epithelial disruption obtained during the $6 \mathrm{~h}$ experiments with culture filtrates of 20 strains of Aspergillus species. Fourteen out of 16 A. fumigatus culture filtrates caused significant slowing of $\mathrm{CBF}(\mathrm{p}<0.01)$ as well as moderate to severe epithelial disruption after $6 \mathrm{~h}$ as compared with the control. Most culture filtrates of A. fumigatus did not slow CBF of human nasal cilia during the first hour of the experiments, except for five culture filtrates (Af-1-Af-3, Af-6 and Af-16). However, subsequently, progressive slowing of $\mathrm{CBF}$ with marked epithelial disruption was observed in these 14 culture filtrates (only the observations after $6 \mathrm{~h}$ are shown in table 2). In 13 A. fumigatus culture filtrates (Af-1-Af-4 and Af-6-Af-14) ciliary dyskinesia was observed in some epithelial strips $1-4 \mathrm{~h}$ after the beginning of experiments, when the ciliary beating was beginning to slow, and in eight A. fumigatus culture filtrates (Af-1-Af-4 and Af6-Af-9) ciliostasis was observed in some areas of the epithelium after 3 to 6 hours incubation. Culture filtrates of $A$. niger and $A$. flavus did not reduce $\mathrm{CBF}$ over $6 \mathrm{~h}$, but all of them caused mild-to-moderate epithelial disruption. On light microscopy, moderate-to-severe epithelial disruption was demonstrated in the epithelial strips after $6 \mathrm{~h}$ incubation at $37^{\circ} \mathrm{C}$ in 18 of 20 culture filtrates of Aspergillus species (Af-1-Af-15, An-1, An2 and Afl-1) (fig. 1A and 1B); although the ciliated epithelium showed intact integrity, or mild disruption at most, after the $6 \mathrm{~h}$ incubation in the control (fig. 1C). In contrast, culture filtrates of clinically isolated $C$. albicans 
Table 2. - In vitro ciliary beat frequency of human respiratory cilia at each experimental time-point (1 and $6 \mathrm{~h}$ ) after exposure to culture filtrates of Aspergillus species

\begin{tabular}{|c|c|c|c|c|}
\hline \multirow{3}{*}{$\begin{array}{l}\text { Strain } \\
\text { No. } \\
\text { Af-1 C.F. }\end{array}$} & \multicolumn{4}{|c|}{ Ciliary beat frequency $\mathrm{Hz}$} \\
\hline & \multicolumn{2}{|l|}{ At $1 \mathrm{~h}$} & \multicolumn{2}{|l|}{ At $6 \mathrm{~h}$} \\
\hline & $8.3(1.6)^{*}$ & ++ & Ciliostasis* & +++ \\
\hline Control & $14.0(1.8)$ & - & $14.4(1.4)$ & + \\
\hline Af-2 C.F. & $10.4(2.2)^{*}$ & + & $2.1(2.0)^{*}$ & +++ \\
\hline Control & $14.0(1.8)$ & - & $14.4(1.4)$ & + \\
\hline Af-3 C.F. & $10.4(2.7)^{*}$ & + & $3.3(4.0)^{*}$ & +++ \\
\hline Control & $14.9(1.7)$ & - & $14.6(1.9)$ & - \\
\hline Af-4 C.F. & $13.0(1.6)$ & + & $3.7(2.5)^{*}$ & +++ \\
\hline Control & $14.0(1.8)$ & - & $14.4(1.4)$ & + \\
\hline Af-5 C.F. & $13.9(1.8)$ & - & $13.4(1.7)$ & ++ \\
\hline Control & $13.7(1.1)$ & - & $13.9(1.7)$ & - \\
\hline Af-6 C.F. & $10.3(2.6)^{*}$ & + & Ciliostasis* & +++ \\
\hline Control & $14.1(1.2)$ & - & $14.0(1.7)$ & - \\
\hline Af-7 C.F. & $13.2(1.4)$ & + & Ciliostasis* & +++ \\
\hline Control & $13.2(1.3)$ & - & $13.1(1.2)$ & - \\
\hline Af-8 C.F. & $12.9(2.0)$ & + & $3.5(3.7)^{*}$ & +++ \\
\hline Control & $13.2(1.3)$ & - & $13.1(1.2)$ & - \\
\hline Af-9 C.F. & $12.8(2.4)$ & + & $5.3(5.3)^{*}$ & +++ \\
\hline Control & $13.7(1.1)$ & - & $13.9(1.7)$ & - \\
\hline Af-10 C.F. & $14.2(1.7)$ & - & $5.9(2.2)^{*}$ & +++ \\
\hline Control & $14.4(1.7)$ & - & $13.8(1.3)$ & - \\
\hline Af-11 C.F. & $15.5(1.5)$ & + & $6.9(4.8)^{*}$ & +++ \\
\hline Control & $14.9(1.9)$ & - & $13.6(1.8)$ & + \\
\hline Af-12 C.F. & $15.3(1.5)$ & + & $8.3(3.4)^{*}$ & +++ \\
\hline Control & $14.9(1.9)$ & - & $13.6(1.8)$ & + \\
\hline Af-13 C.F. & $13.1(1.9)$ & - & $7.9(3.7)^{*}$ & +++ \\
\hline Control & $14.1(2.0)$ & - & $12.4(1.4)$ & - \\
\hline Af-14 C.F. & $12.9(1.2)$ & - & $9.9(1.8)^{*}$ & ++ \\
\hline Control & $14.1(2.0)$ & - & $12.4(1.4)$ & - \\
\hline Af-15 C.F. & $12.1(1.4)^{*}$ & - & $11.8(1.7)^{*}$ & ++ \\
\hline Control & $14.1(1.2)$ & - & $14.0(1.2)$ & - \\
\hline Af-16 C.F. & $13.2(1.8)$ & - & $13.0(1.9)$ & + \\
\hline Control & $14.0(0.8)$ & - & $14.4(1.4)$ & + \\
\hline An-1 C.F. & $12.7(1.6)$ & - & $12.2(1.5)$ & ++ \\
\hline Control & $13.2(1.3)$ & - & $13.2(1.2)$ & - \\
\hline An-2 C.F. & $13.1(1.8)$ & - & $12.1(1.7)$ & ++ \\
\hline Control & $13.9(2.3)$ & - & $12.6(1.0)$ & - \\
\hline An-3 C.F. & $13.2(1.1)$ & - & $12.6(1.5)$ & + \\
\hline Control & $13.2(1.3)$ & - & $13.1(1.2)$ & - \\
\hline Afl-1 C.F. & $12.5(1.5)$ & - & $12.5(1.5)$ & ++ \\
\hline Control & $13.9(2.3)$ & - & $12.6(1.0)$ & - \\
\hline
\end{tabular}

Values are represented as mean (SD) $(n=10)$. C.F.: culture filtrate; Control: Medium 199. Degree of disruption of ciliated epithelium is indicated as follows; - : intact; +: undulation of the epithelial surface; ++: undulation and extrusion of individual cells from the epithelial surface; +++: total disorganization of the epithelial surface. *: $\mathrm{p}<0.01$ with reference to control value. Af: A. fumigatus; An: A. niger; Afl: A. flavus.

and $\mathrm{Cr}$. neoformans had no effects on CBF but, in only one strain, caused mild disruption to surface integrity of respiratory epithelium, which was similar to that shown in control experiments with Medium-199 after the $6 \mathrm{~h}$ incubation (table 3).
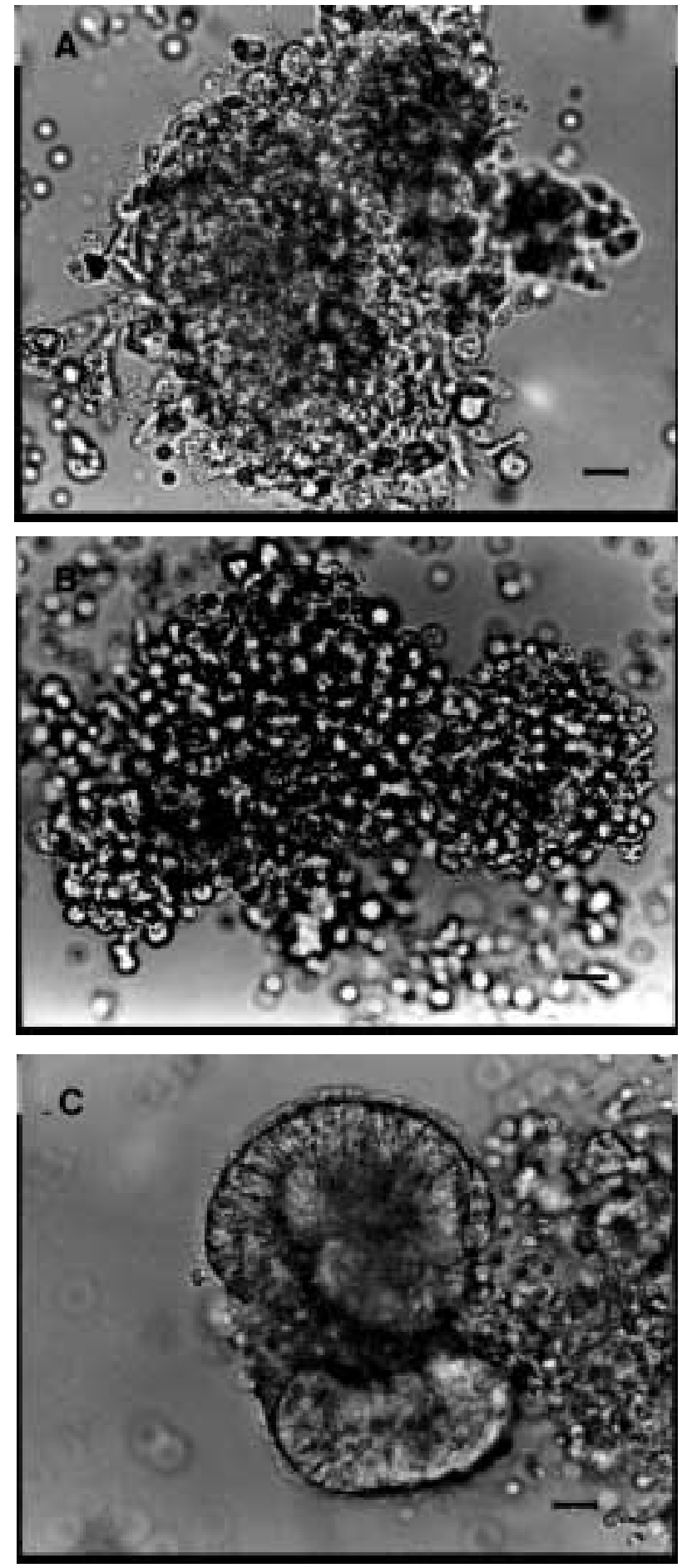

Fig. 1. - The effect of culture filtrates of A. fumigatus on human nasal ciliated epithelium assessed by light microscopy. After $6 \mathrm{~h}$ experiments, A) ciliated epithelium showed moderate disruption (++) in the culture filtrate of Af-14; B) ciliated epithelium also showed severe disruption (+++) in Af-6 culture filtrate; C) in comparision the epithelium is intact $(-)$ in control fluid (Medium-199). (Original magnification $\times$ 400; internal scale bars $=25 \mu \mathrm{m})$.

The effects of sputum sols on respiratory ciliated epithelium

Figure 2 shows the mean $(n=10) C B F$ values measured throughout the $6 \mathrm{~h}$ experiments with the five sputum sols 
Table 3. - In vitro ciliary beat frequency of human respiratory cilia at each experimental time-point (1 and $6 \mathrm{~h}$ ) after exposure to culture filtrates of $C$. albicans and $C r$. neoformans

\begin{tabular}{lcccc}
\hline \multirow{2}{*}{$\begin{array}{l}\text { Strain } \\
\text { No. }\end{array}$} & \multicolumn{5}{c}{ Ciliary beat frequency Hz } \\
At $1 \mathrm{~h}$ & At $6 \mathrm{~h}$ \\
\hline Ca-1 C.F. & $14.6(1.6)$ & - & $13.1(1.5)$ & - \\
Control & $13.6(1.7)$ & - & $13.1(2.1)$ & - \\
Ca-2 C.F. & $13.0(1.6)$ & - & $14.2(1.5)$ & + \\
$\quad$ Control & $14.2(2.2)$ & - & $14.3(2.0)$ & - \\
Ca-3 C.F. & $14.0(1.9)$ & - & $14.5(2.5)$ & - \\
$\quad$ Control & $14.1(1.2)$ & - & $12.6(1.4)$ & - \\
Cr-1 C.F. & $13.5(2.1)$ & - & $13.2(2.1)$ & - \\
$\quad$ Control & $13.6(1.7)$ & - & $13.1(2.1)$ & - \\
Cr-2 C.F. & $13.7(1.5)$ & - & $14.1(1.5)$ & - \\
Control & $14.2(2.2)$ & - & $14.3(2.0)$ & - \\
& & & & \\
\hline
\end{tabular}

Values are represented as mean (SD) $(n=10)$. For symbols and abbreviations see legend to table 2 .

from patients with pulmonary aspergillosis (two with CNPA and three with pulmonary aspergilloma) infected by A. fumigatus. Of 20 patients, most expectorated only a small quantity of sputum, hence enough sputum for experiments was obtained from only five patients (Nos. 1-5). Appearance of the sputum was mucoid or mucopurulent, but the purulent portion was less than one third of the whole sputum. All of the five sputum sols caused significant $(\mathrm{p}<0.01)$ slowing of $\mathrm{CBF}$ associated with moderate to severe epithelial disruption during the $6 \mathrm{~h}$ experiments, in comparison with both Medium-199 and the sputum sol from the bronchiectatic patient (No. P-1) infected by $P$. aeruginosa. The changes observed in $\mathrm{CBF}$ and the epithelial structure were of a magnitude similar to those caused by culture filtrates of A. fumigatus. Cilioinhibition was beginning to occur during the first $2 \mathrm{~h}$ of the experiments, and continued slowing of CBF was observed throughout the $6 \mathrm{~h}$ experiments. All the sputum sols from the patients with pulmonary aspergillosis also caused ciliary dyskinesia in some areas of the epithelial strips during the last $4 \mathrm{~h}$ of the experiments, and two sputum sols (Nos. 1 and 4) caused ciliostasis in some epithelial strips after $6 \mathrm{~h}$ incubation. Whereas, the sputum sol from the bronchiectatic patient (No. P1) infected by $P$. aeruginosa caused mild epithelial disruption, but did not slow $\mathrm{CBF}$ throughout the $6 \mathrm{~h}$ experiments.

In another experiment using the same method, three sputum sols obtained from bronchiectatic patients (Nos. P-2-4) infected by $P$. aeruginosa caused mild (Nos. P2 and P-3) to moderate (No. P-4) epithelial disruption, but did not slow CBF significantly during the $6 \mathrm{~h}$ experiments; P-2 sputum sol (at 1 and $6 \mathrm{~h}$ ): 13.4 and 13.0 $\mathrm{Hz}$; P-3 sputum sol 13.6 and $12.7 \mathrm{~Hz}$; and P-4 sputum sol 13.3 and $12.3 \mathrm{~Hz}$, respectively; whereas, two sputum sols from pulmonary aspergillosis (Nos. 1 and 5) caused significant CBF slowing with severe epithelial disruption; No. 1 (at 1 and $6 \mathrm{~h}$ ) 12.8 and $5.2 \mathrm{~Hz}$; and No. 513.2 and $9.5 \mathrm{~Hz}$, respectively; and Medium-199 (control) had no effects on ciliated epithelium in the same experiment.

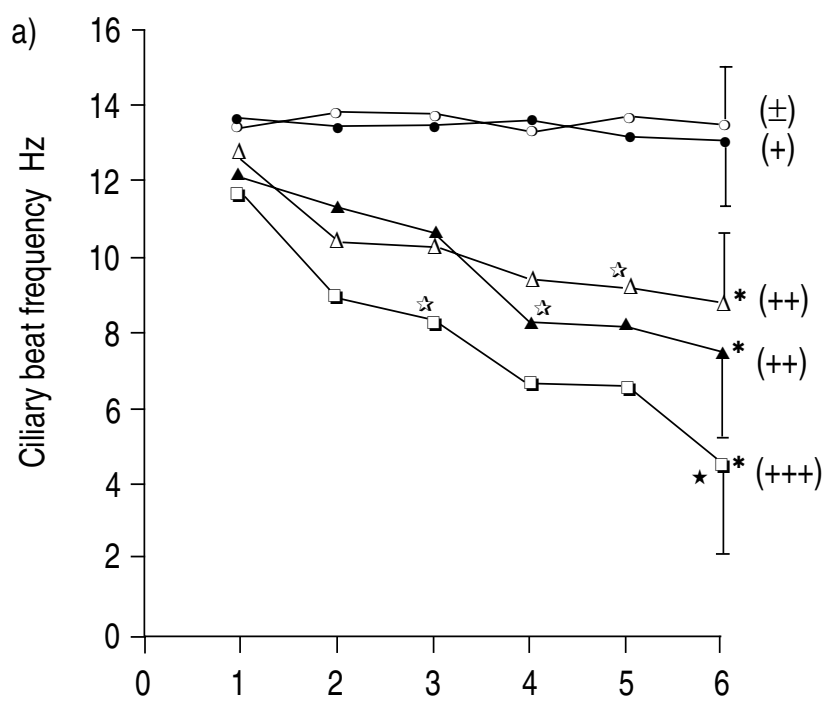

Time $h$

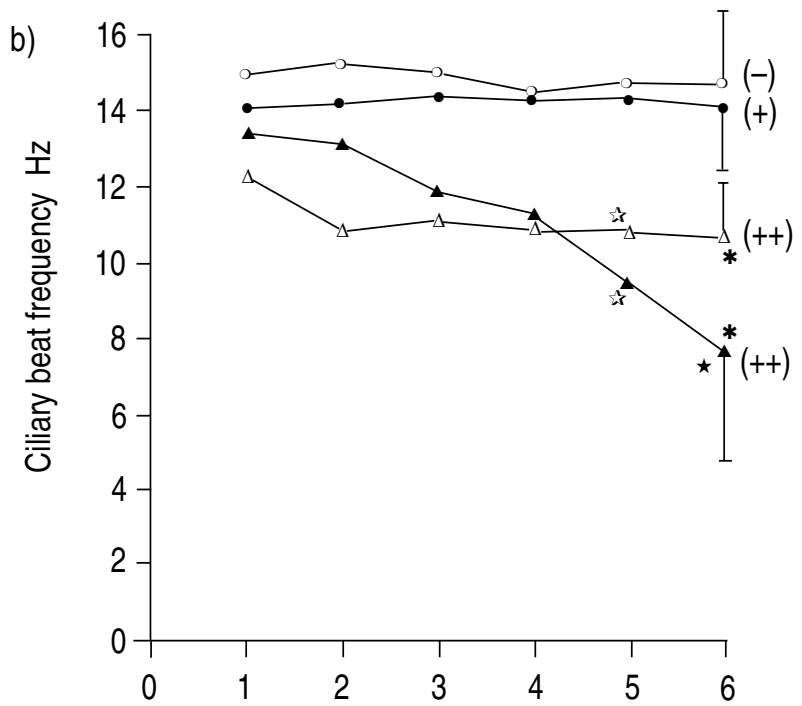

Time $h$

Fig. 2. - Mean values of ciliary beat frequency (CBF) measured throughout $6 \mathrm{~h}$ experiment with sputum sols from a) patients (Nos. 1, 3 and 5) with pulmonary aspergillosis and from a patient (No. P-1) with bronchiectasis infected by $P$. aeruginosa. The mean CBF values are shown as fol-

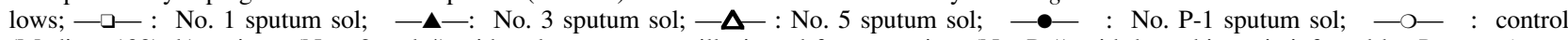
(Medium-199). b) patients (Nos. 2 and 4) with pulmonary aspergillosis and from a patient (No. P-1) with bronchiectasis infected by $P$. aeruginosa.

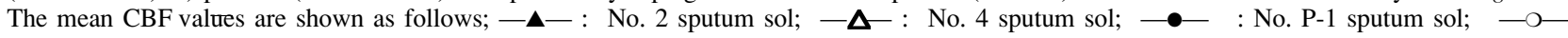
: control (Medium-199). *: $\mathrm{p}<0.01$ with reference to values obtained in control (Medium-199) as well as in the the sputum sol from the bronchiectacic patient (No. P-1). The degree of epithelial disruption is indicated in parenthesis as described in Table 2 . $i x$ : ciliary dyskinesia first observed; $\star$ : ciliostasis first observed. Bars represent 1 SD. 


\section{Discussion}

We demonstrated in the present in vitro study that most of the culture filtrates of clinically isolated A fumigatus affected human respiratory ciliated epithelium by slowing CBF significantly and disrupting epithelial integrity during the $6 \mathrm{~h}$ period at $37^{\circ} \mathrm{C}$, in comparison with the control fluid (Medium-199), and that culture filtrates of A. niger and A. flavus also disrupted human respiratory epithelium without significant $\mathrm{CBF}$ slowing. On the other hand, we did not demonstrate any effects of culture filtrates of other clinically isolated pathogenic fungi, $C$. albicans (three strains) and $\mathrm{Cr}$. neoformans (two strains), on human respiratory ciliated epithelium throughout the $6 \mathrm{~h}$ experiments using the same methods.

Sputum sols from pulmonary aspergillosis infected by A. fumigatus also caused significant slowing of CBF associated with epithelial disruption of human respiratory epithelium in vitro, in comparison with sputum sol from a bronchiectatic patient infected by $P$. aeruginosa.

Sputum consists of both host- and parasite-derived components, and the host-derived neutrophil elastase has been known to be capable of damaging respiratory epithelial [16, 22-24]. AMITANi et al. [16] reported that human neutrophil elastase caused damage to human respiratory ciliated epithelium at $100-500 \mu \mathrm{g} \cdot \mathrm{mL}^{-1}$ and slowed CBF at $500 \mu \mathrm{g} \cdot \mathrm{mL}^{-1}$ in vitro. Nevertheless, the effects of the sputum sols seem to be shared in greater part by the Aspergillus-derived component, because all sputum samples obtained from pulmonary aspergillosis in this study were all mucoid or mucopurulent, with the purulent portion less than one third, and because the sputa with less cilio-inhibitory activity from bronchiectatic patients infected by $P$. aeruginosa had yellowish-green appearance, which almost invariably suggested a higher concentration of neutrophil elastase [25].

Consequently, the results of the present study support our speculation that Aspergillus species may release factor(s) which cause damage to human respiratory ciliated epithelium, possibly to protect themselves from elimination by mucociliary clearance and to persist in the site of their colonization of the lower respiratory tracts of patients with underlying bronchopulmonary conditions which have already affected the clearing system to some extent.

There have been many reports on Aspergillus colonization in the lower respiratory tracts and the lungs of patients with localized underlying bronchopulmonary conditions, including healed tuberculous cavities, cystic fibrosis $(\mathrm{CF})$, bronchiectasis and patients with ABPA, where local host defence, especially mucociliary clearance, is supposed to be already impaired [2-9]. Also, in patients with CF or bronchiectasis of other causes, some species of bacteria, including $P$. aeruginosa, St. Pneumoniae, and $H$. influenzae, more frequently colonize the lower respiratory tracts as a result of the impairment of mucociliary clearance of the airways. Many studies have been carried out on the mechanisms of the bacterial colonization and the pathogenesis of chronic infections in the lower respiratory tracts, and some cilio-inhibitory substances, produced by $P$. aeruginosa, St. pneumoniae, and $H$. influenzae have already been isolated [14-18,
26]. It is well-known that $P$. aeruginosa, in particular, releases several cilio-inhibitory substances, such as phenazine pigments (pyocyanin, 1-hydroxyphenazine) [14, 26], haemolysin (rhamonolipid) [15], and at least two kinds of proteinases (elastase, alkaline protease) [16, 27-29]. It is also known that human neutrophil elastase causes damage to the host tissues, including respiratory ciliated epithelium, as well as to pathogenic micro-organisms [16, 23]. It has been suggested that these substances, derived both from bacteria and host defence itself, may play important roles in the development of the colonization and subsequent obstinate chronic infections of the bacteria in the lower respiratory tracts by disturbing ciliary movement, disrupting the integrity of the epithelium, and stimulating mucus production, all of which may create a favourable environment for bacterial proliferation $[10,11]$.

In addition to the bacteria, Aspergillus species, by itself or in combination with bacteria, can colonize the lower respiratory tracts with localized underlying disorders where other pathogenic fungi rarely colonize. The reasons why, among many pathogenic fungi, Aspergillus species almost exclusively colonize the lower respiratory tracts are not yet known.

Although there have been no reports, to our knowledge, on the direct effects of Aspergillus species on human respiratory ciliated epithelium, the only one report related to the epithelial damage caused by A. fumigatus extract was made by RoBinson et al. [30] in 1990. They investigated the effects of A. fumigatus extract obtained by sonication of the mycelium on human amniotic epithelium (not respiratory epithelium), to gain insight into the significance of Aspergillus-derived substances in producing marked bronchial damage, which may lead to proximal bronchiectasis, known to be one of the characteristic features in ABPA. In the study, they revealed the ability of $A$. fumigatus extract to induce epithelial cell detachment from the basement membrane, and also revealed the presence of a proteinase in the A. fumigatus extract. They concluded that the bronchial damage in ABPA may be directly mediated by A. fumigatus-derived products, including a chymotrypsin-like proteinase, as well as by immunological mechanisms, particularly a type III reaction within the airway wall. Since they adopted an in vitro model consisting of human amniotic cells and native basement membrane as the target tissue, it is urgently required that corresponding experiments using human respiratory ciliated epithelium should be performed before any extrapolation can be made to human respiratory tract disease.

In the present study, we investigated, for the first time, the effects of the culture filtrates of Aspergillus species and sputum sols from patients with pulmonary aspergillosis on human respiratory ciliated epithelium, and demonstrated ciliary beat slowing and epithelial damage in vitro. This suggests that Aspergillus species, especially A. fumigatus, release a factor (or factors) that slows ciliary movement, disorganizes their beating, and disrupts epithelial integrity. It is, therefore, tempting to speculate that human respiratory epithelium in vivo might be similarly affected by Aspergillus products. This property of Aspergillus species should be important in establishing the colonization 
and infectious process by rendering mucociliary clearance less efficient than ever, thus allowing the organism to multiply easily in the airways and the lungs. It might also assist in the perpetuation and spread of existing infection within the airways and the lungs, thereafter.

The cilio-inhibitory activity of culture filtrates of $A$. fumigatus isolated from three patients with both systemic and localized compromised host defences were also detected. The significance of the cilio-inhibitory activity of Aspergillus species in occurrence of invasive aspergillosis, especially in the systemic compromised host cannot be elucidated in the present study; however, it is likely that the activity may play a role even in the development of invasive aspergillosis in the systemic compromised host.

In future studies, isolation and characterization of the cilio-inhibitory factor(s) will be beneficial in order to clarify the pathogenetic mechanisms of Aspergillus colonization in the lower respiratory tracts, and also the mechanisms of the fungal spread in pulmonary aspergillosis.

Acknowledgements: The authors wish to thank T. Homma and $\mathrm{T}$. Ueda, Department of Infection and Inflammation, and $\mathrm{K}$. Maeda, Clinical Laboratory, Chest Disease Research Institute, Kyoto University, for their kind assistance in culturing of the fungi. The authors appreciate the assistance of K. Kataoka in the preparation of this manuscript.

\section{References}

1. Comstock GW, Palmer CE, Stone RW, Goodman NL. Fungi in the sputum of normal men. Mycopathol Mycol Appl 1974; 54: 55-62.

2. The Research Committee of the British Thoracic and Tuberculosis Association. Aspergilloma and residual tuberculosis cavities: the results of a resurvey. Tubercle 1970; 51: 227-245.

3. Tomlinson JR, Sahn SA. Aspergilloma in sarcoid and tuberculosis. Chest 1987; 92: 505-508.

4. Nelson LA, Callerame ML, Schwartz RH. Aspergillosis and atopy in cystic fibrosis. Am Rev Respir Dis 1979; 120: 863-873.

5. Mroueh S, Spock A. Allergic bronchopulmonary aspergillosis in patients with cystic fibrosis. Chest 1994; 105 : 32-36.

6. Orie NGM, de Vries GA, Kikstra A. Growth of Aspergillus in the human lung: aspergilloma and aspergillosis. Am Rev Respir Dis 1960; 82: 649-662.

7. Smith FB, Debra Beneck. Localized Aspergillus infestation in primary lung carcinoma: clinical and pathological contrasts with post-tuberculous intracavitary aspergilloma. Chest 1991; 100: 554-556.

8. McConnochie K, O'Sullivan M, Khalil JF, Pritchard MH, Cibbs AR. Aspergillus colonization of pulmonary rheumatoid nodule. Respir med 1989; 83: 157-160.

9. Safirstein BH. Aspergilloma consequent to allergic bronchopulmonary aspergillosis. Am Rev Respir Dis 1973; 108: 940-943.

10. Cole PJ. A new look at the pathogenesis and management of persistent bronchial sepsis: a "vicious circle" hypothesis and its logical therapeutic connotations. In: Davis RJ, ed. Strategies For the Management of Chronic Bronchial Sepsis. Oxford, The Medicine Publishing Foundation, 1984; pp. 1-20.

11. Cole PJ, Wilson R. Host-microbial interrelationships in respiratory infection. Chest 1989; 95: 217S-221S.
12. Wilson R, Roberts D, Cole P. Effect of bacterial products on human ciliary function in vitro. Thorax 1985; 40: 125-131.

13. Hingley ST, Hastie AT, Kueppers F, Higgins ML, Weinbaum G, Shryock T. Effect of ciliostatic factors from Pseudomonas aeruginosa on rabbit respiratory cilia. Infect Immun 1986; 51: 254-262.

14. Wilson R, Pitt T, Taylor G, et al. Pyocyanin and 1hydroxyphenazine produced by Pseudomonas aeruginosa inhibit the beating of human respiratory cilia in vitro. $J$ Clin Invest 1987; 79: 221-229.

15. Wilson R, Hastie A, Munro N, et al. Effect of rhamnolipid from Pseudomonas aeruginosa on human ciliated epithelium in vitro and on in vivo mucociliary transport in an animal model. Am Rev Respir Dis 1988; 137 (Suppl.): 171

16. Amitani R, Wilson R, Rutman A, et al. Effects of human neutrophil elastase and Pseudomonas aeruginosa proteinases on human respiratory epithelium. Am J Respir Cell Mol Biol 1991; 4: 26-32.

17. Steinfort C, Wilson R, Mitchell T, et al. Effects of Streptococcus pneumoniae on human respiratory epithelium in vitro. Infect Immun 1989; 57: 2006-2013.

18. Read RC, Rutman AA, Jeffery PK, et al. Interaction of capsulate Haemophilus influenzae with human airway mucosa in vitro. Infect Immun 1992; 60: 3244-3252.

19. Rutland J, Cole P. Noninvasive sampling of nasal cilia for measurement of beat frequency and study of ultrastructure. Lancet 1980; ii: 564-565.

20. Binder RE, Faling LJ, Pugatch RD, Mahasaen C, Snider GL. Chronic necrotizing pulmonary aspergillosis: a discrete clinical entity. Medicine 1982; 61: 109-124.

21. Hinson KFW, Moon AJ, Plummer NS. Bronchopulmonary aspergillosis: a review and a report of eight new cases. Thorax 1952; 7: 317-333.

22. Tegner H, Ohlsson K, Toremalm NG, von Mecklenburg C. Effect of human leukocyte enzymes on tracheal mucosa and its mucociliary activity. Rhinology 1979; 17: 199-206.

23. Smallman LA, Hill SL, Stockley RA. Reduction of ciliary beat frequency in vitro by sputum from patients with bronchiectasis: a serine proteinase effect. Thorax 1984; 39: 663-667.

24. Sykes DA, Wilson R, Creenstone M, Currie DC, Steinfort C, Cole PJ. Deleterious effects of purulent sputum sol on human ciliary function in vitro: at least two factors identified. Thorax 1987; 42: 256-261.

25. Stockley RA, Hill SL, Morrison HM, Starkie CM. Elastolytic activity of sputum and its relation to purulence and to lung function in patients with bronchiectasis. Thorax 1984; 39: 408-413.

26. Watson D, MacDermot J, Wilson R, Cole PJ, Taylor GW. Purification and structural analysis of pyocyanin and 1-hydroxyphenazine. Eur J Biochem 1986; 159: 309-313.

27. Morihara K, Tsuzuki H. Effect of cobalt ion on the enzymatic activity of Pseudomonas aeruginosa alkaline proteinase. Agr Biol Chem 38: 1974; 621-626.

28. Morihara K, Tsuzuki H. Psudomonas aeruginosa elastase: affinity chromatography and some properties as a metallo-neutral proteinase. Agr Biol Chem 1975; 39: $1123-1128$.

29. Morihara K, Homma JY. Pseudomonas proteases. In: Holder IA, ed. Bacterial enzymes and virulence. Boca Raton, Florida: CRC Press, Inc., 1985; pp. 41-79.

30. Robinson BWS, Venaille TJ, Mendis AHW, McAleer R. Allergens as proteases: an Aspergillus fumigatus proteinase directly induces human epithelial cell detachment. J Allergy Clin Immunol 1990; 86: 726-731. 